\title{
Motivações para realizar práticas de cuidado na gestação de alto risco
}

\author{
Motivations to perform care practices in high-risk pregnancy \\ Motivaciones para realizar las prácticas de atención en embarazo de alto riesgo
}

Recebido: 27/12/2020 | Revisado: 03/01/2021 | Aceito: 05/01/2021 | Publicado: 06/01/2021

Laís Antunes Wilhelm

ORCID: https://orcid.org/0000-0001-6708-821X Universidade Federal de Santa Catarina, Brasil

E-mail: lais.wilhelm@ufsc.br

Lisie Alende Prates

ORCID: https://orcid.org/0000-0002-5151-0292 Universidade Federal do Pampa, Brasil

E-mail: lisiealende@hotmail.com

Camila Neumaier Alves

ORCID: https://orcid.org/0000-0002-6455-3689

Centro Universitário Ritter dos Reis, Brasil

E-mail: camilaenfer@gmail.com

Juliane Scarton

ORCID: https://orcid.org/0000-0002-3676-0672

Universidade Federal de Rio Grande, Brasil

E-mail: juliscarton10@hotmail.com

Luiza Cremonese

ORCID: https://orcid.org/0000-0001-7169-1644 Universidade Luterana do Brasil, Brasil E-mail: lu_cremonese@ @otmail.com

\begin{abstract}
Resumo
Objetivo: conhecer as práticas de cuidado realizadas por gestantes de alto risco para preservar a gravidez e as motivações para realizá-las. Método: pesquisa qualitativa, realizada com dez mulheres que vivenciaram a gestação de alto risco. Os dados foram produzidos por meio de entrevista semiestruturada e submetidos à análise de conteúdo temática. A pesquisa seguiu os aspectos éticos, de acordo com as normas da resolução no $466 / 12$, sendo aprovada pelo parecer 222.320. Resultados: as motivações para as práticas de cuidado realizadas pelas gestantes visavam garantir a saúde do bebê e englobavam cuidados com a alimentação, o repouso e o afastamento de fatores estressantes. Eram práticas advindas de conhecimentos informais e profissionais. Conclusão: as gestantes de alto risco realizavam cuidados que envolviam demandas de diferentes esferas de necessidades e demonstravam necessidade de apoio emocional devido às mudanças ocorridas em sua rotina e no seu estilo de vida.
\end{abstract}

Palavras-chave: Gravidez de alto risco; Saúde da mulher; Cuidado pré-natal.

\begin{abstract}
Objective: to know the care practices performed by high-risk pregnant women to preserve pregnancy and the motivations to perform them. Method: qualitative research conducted with ten women who experienced high-risk pregnancy. The data were produced through semi-structured interviews and submitted to thematic content analysis. The research followed the ethical aspects, according to the norms of resolution number 466/12, being approved by opinion 222.320. Results: the motivations for the care practices performed by the pregnant women aimed to ensure the baby's health and encompassed care with food, rest and the removal of stressful factors. They were practices departing from informal and professional knowledge. Conclusion: high-risk pregnant women performed care that involved demands from different spheres of needs and demonstrated the need for emotional support due to changes in their routine and lifestyle.
\end{abstract}

Keywords: Pregnancy, high-risk; Women's health; Prenatal care.

\section{Resumen}

Objetivo: conocer las prácticas de cuidado realizado por las mujeres embarazadas de alto riesgo para la preservación de embarazo y las motivaciones para llevar a cabo los. Método: investigación cualitativa, se realizó con diez mujeres que han experimentado el embarazo de alto riesgo. Recolección de datos produjo a través de la entrevista semiestructurada, se utiliza como análisis de datos el análisis de contenido temático. La investigación siguió los aspectos éticos, de acuerdo com las normas de la resolución 466/12, aprobada por el dictamen 222.320. Resultados: las motivaciones para las prácticas de cuidado llevadas a cabo por las mujeres embarazadas tenían como objetivo garantizar la salud del bebé e incluían el cuidado con alimentos, descanso y la eliminación de factores estresantes. Estas prácticas provienen del conocimiento informal y profesional. Conclusión: las mujeres embarazadas en el 
cuidado de alto riesgo celebraron que implican demandas para diferentes niveles de exigencia y necesidad de apoyo emocional debido a los cambios en su rutina y su estilo de vida.

Palabras clave: Embarazo de alto riesgo; Salud de la mujer; Atención prenatal.

\section{Introdução}

A gestação e o parto são processos naturais na vida feminina e, geralmente, têm desfecho bem-sucedido. Porém, em algumas circunstâncias, fatores relativos à assistência inadequada e/ou associados a problemas prévios vivenciados pela mulher, como doenças preexistentes, podem culminar em óbito ou sequelas para a mãe e o concepto, tornando a gestação com risco obstétrico e/ou fetal (Lima et al., 2015).

Para a atenção adequada à gestante de alto risco, há de se considerar o conhecimento de suas práticas de cuidado, as quais representam, neste estudo, práticas sociais definidas historicamente que retratam valores e princípios de um determinado grupo (Lima et al., 2015). Estas ocorrem nas relações sociais e se estabelecem por meio da aproximação dos saberes populares com os científicos, havendo, portanto, a necessidade de coexistência entre os procedimentos convencionais e não convencionais.

Deste modo, é importante que o profissional de saúde conheça e valorize as práticas de cuidado realizadas pela gestante de alto risco para que possa planejar uma atenção contextualizada a cada mulher. Ademais, destaca-se que a gestação é um período em que há alterações fisiológicas e psicológicas que demandam necessidades específicas naturalmente, reforçando a prioridade de conhecer como essas mulheres cuidam de sua saúde e como são seus hábitos, crenças e rotinas familiares (Ortigara, Carvalho \& Pelloso, 2015).

Ao conhecer estas práticas, é possível trocar, horizontalmente, orientações e percepções acerca do cuidado realizado. Nesta condição, o enfermeiro tem oportunidade de exercer seu papel de cuidador e educador, dividindo o seu saber, evitando, assim, posturas autoritárias. Pela aproximação com a gestante, há maior chance de adesão e efetivo cuidado (Rodrigues et al., 2016). Na gestação de alto risco, há demanda acrescida de cuidados especiais, produção de sentimentos relativos ao risco da gravidez, tanto para ela quanto para o concepto, assim como maior probabilidade de alteração da rotina da mulher e de sua família, o que a sobrecarrega emocionalmente (Wilhelm et al., 2015; Oliveira \& Mandú, 2015).

A gravidez de alto risco representa de 10 a 20\% das gestações no Brasil (Ricci, 2015). De 1990 para 2013, o número de mortes maternas reduziu de 120 para 69 óbitos por 100.000 nascidos vivos, representando uma queda de $43 \%$ na razão de mortalidade materna. Apesar disso, o Brasil não atingiu o quinto objetivo de desenvolvimento do milênio (ODM), revelando que a maioria desses óbitos poderia ser evitado se as gestantes fossem amparadas há tempo (Resende, Rodrigues \& Fonseca, 2015). Ratifica-se então, que as ações tomadas em 2015 em relação aos ODM, resultaram nos novos Objetivos de Desenvolvimento Sustentável (ODS). Nesse sentido, a nova meta até 2030, no Brasil, é reduzir a taxa de mortalidade materna para no máximo 30 mortes por 100.000 nascidos vivos e para a Taxa de Mortalidade Neonatal foi estimada uma redução de 43,7\%, que aponta para uma taxa de mortalidade de 5,3 por 1.000 nascidos vivos (Brasil, 2018), a qual está, muitas vezes, relacionada com a saúde materna.

Frente a essa realidade, percebe-se a relevância de um cuidado atento, durante o período gravídico-puerperal, justificando, assim, a relevância deste estudo, o qual teve como objetivo conhecer as práticas de cuidado realizadas pelas gestantes de alto risco para manter a sua gravidez e o que as motivou a realizá-las. Este estudo tem como questão de pesquisa: quais as práticas de cuidado vivenciadas por mulheres que tiveram uma gestação de alto risco e quais foram as motivações para realizá-las? 


\section{Metodologia}

Trata-se de estudo derivado de dissertação de mestrado. A pesquisa é de natureza qualitativa do tipo descritiva, na qual foi utilizada a entrevista semiestruturada como técnica de coleta de dados. As entrevistas foram realizadas nos meses de maio a setembro de 2013, após a aprovação do projeto de pesquisa pelo Comitê de Ética em Pesquisa, sob Certificado de Apresentação para Apreciação Ética sob no 13178713.3.0000.5346 e parecer número 222.320, com data de aprovação 19 de março de 2013. A pesquisa seguiu os preceitos da Resolução no. 466/12, do Conselho Nacional de Saúde, do Ministério da Saúde.

Participaram do estudo dez mulheres, as quais foram captadas após a busca em prontuários do Ambulatório de um Hospital de ensino do Sul do país, local onde ocorriam as consultas de pré-natal de alto risco. Os critérios de inclusão envolveram mulheres com idade superior a 18 anos, consideradas gestantes de alto risco e que o parto tivesse ocorrido no máximo há dois anos, na tentativa de evitar viés de memória. Os critérios de exclusão consideraram as mulheres que não realizaram todo o pré-natal no referido hospital.

Os dados foram submetidos à análise de conteúdo temática da proposta operativa, caracterizada por dois momentos operacionais. O primeiro corresponde às deliberações fundamentais da pesquisa, delineado na fase exploratória da investigação. O segundo, denominado de interpretativo, foi balizado pelo encontro com os fatos empíricos, que se dividiu em duas etapas: ordenação e a classificação dos dados (Minayo, 2014).

\section{Resultados}

Durante a gravidez, a mulher passa por diversas mudanças de ordem biológica e emocional, ocasionando algumas demandas específicas de cuidado. Neste sentido, os resultados do estudo englobam as práticas de cuidado de mulheres que vivenciaram a gestação de alto risco e as motivações que levaram estas a realizá-las.

A alimentação saudável emergiu como uma prática de cuidado realizada pelas mulheres que vivenciaram a gestação de alto risco. Foi motivada por ser uma maneira de não agravar o risco gestacional ou desenvolver outra situação que pudesse complicar a gravidez.

Tive que tomar cuidado com a minha alimentação, para não ganhar mais peso do que já tinha, porque eu já tenho o problema da obesidade e também para não desenvolver a diabetes, né?! Eu comia saladinha, meu arrozinho e a minha carninha magrinha com a maior felicidade (E2).

Eu sabia que tinha que tomar os meus cuidados, né?! Na questão da alimentação, eu comecei a me cuidar desde o momento que fiquei sabendo (que era gestante de alto risco), por conta própria, mas depois a nutricionista me acompanhou. A minha alimentação alterou muito, nada de sal, por causa da hipertensão e também mudou bastante por causa da diabetes gestacional. Eu comia só coisas integrais, frutas, verduras e era tudo pouquinho, e mais vezes durante o dia. Eu perdi dez quilos durante a gestação, isso me ajudou bastante (E3).

Elas (médica e enfermeira) me diziam que eu tinha que me alimentar, porque era muito magrinha. Diziam para eu comer feijão e frutas, essas coisas, porque tive anemia. Aí comecei comer toda hora e tomar bastante água (E5). 
Outra prática de cuidado realizada pelas mulheres foi o repouso, o qual alterou o cotidiano e os afazeres, e foi considerado por algumas como algo difícil. Algumas precisaram pedir afastamento do emprego, não podiam realizar esforço físico, ou precisaram abster-se das relações sexuais neste período.

Não foi fácil, em casa eu não podia fazer nada, era só deitada, sentada, fazendo repouso, ficava angustiada pensando no que tinha para fazer, tendo quatro filhos para cuidar (E1).

A gente faz muito esforço, lá onde eu trabalho. Então, no final das contas, eu tive que me afastar do serviço, o que foi bem difícil no começo, porque eu sempre tive uma vida bem corrida, mas depois me acostumei. Estava proibido durante toda a gestação, tudo que envolvesse qualquer esforço físico. Ficou meio complicado pelo lado do meu casamento, porque meu marido é jovem, né?! Somos casados há pouco tempo e a médica proibiu qualquer tipo de relação sexual e isso mexe com o emocional da gente, até porque eu acho que, durante a gestação, a gente fica insegura (E4).

Tive que fazer repouso, principalmente no último mês. Não queria ficar sem fazer nada, mas tinha que ficar em repouso, fazer o que, precisei me motivar para o bem do bebê, né?! (E6).

Com 30 semanas, internei para segurar o bebê. Depois daquilo eu não pude mais trabalhar, fiquei angustiada. Eles (médico e enfermeiro) me diziam para ficar em repouso e não fazer nenhum esforço fisico. Até para fazer relação (sexual) o doutor proibiu. Tive que me cuidar e pensar bastante no bebê para conseguir ficar quieta e não realizar nenhum esforço físico (E9).

Também é importante considerar os aspectos emocionais relativos à vivência de uma gestação de alto risco, pois envolve uma ameaça clara à vida da gestante e/ou do concepto. Logo, a atenção precisa ser redobrada, para que isso não se torne mais um agravante nesse período. Assim, verificou-se que as relações interpessoais podem interferir nos aspectos emocionais das gestantes de alto risco, sendo relatado, como uma prática de cuidado, evitar situações estressantes com pessoas próximas.

Eu evitava ao máximo não me estressar com meus colegas, porque eu tinha problema com um colega e, até mesmo, com a família. Nunca tive estresse, nem nada, mas eu evitava ao máximo qualquer coisa que me afetasse. Sabia que ele (bebê) precisava de mim, não queria que nada passasse para ele (E10).

Ao contrário dos demais depoimentos, uma entrevistada relatou que não realizava nenhum cuidado. Nas gestações anteriores, que também eram de alto risco, ela menciona que teve mais cuidados.

Olha, eu vou ser bem sincera, não realizei nenhum cuidado especial, porque dos outros (filhos/gestações) eu me cuidei, me cuidei e vivia dentro do hospital e não gostava de lá. Nas outras gestações, eu não limpava a casa. Era minha mãe que limpava. Meи ex-marido pagava empregada. Eu não fazia nada e estava sempre doente. Nessa gravidez eu não tinha condições financeiras para pagar alguém. Sou caprichosa. Então, fazia o serviço de casa. Eu não me sentia mal. Não tinha por que fazer repouso. Então, não me cuidei como era para me cuidar, como fiz nas outras vezes (E7). 
Durante as entrevistas, as mulheres também foram questionadas sobre o que as motivou a realizar as práticas de cuidado durante a gestação de alto risco. Os relatos estavam relacionados ao bem-estar seu ou do bebê para poder educar, cuidar e criar os filhos.

Eu estava tão focada e preocupada no bem-estar do bebê que estava disposta a fazer o que fosse necessário. Eu sabia dos cuidados que tinha que ter e fazia com maior prazer. Pensava só em coisas boas, porque sabia que era uma coisa que ia levar para o resto da minha vida, sabia que ia valer a pena. O que são nove meses para depois ter um filho para o resto da vida?! (E2).

Tenho que levar essa gestação até o fim. Coloquei na minha cabeça que era pro bem do bebê, pensava só nisso (E4).

Eu pensava que tinha que tomar cuidado, né?! Meu médico me disse: ou tu tomas cuidado para criar teus cinco filhos, ou tu faz as coisas e não vê nenhum deles se criar. Daí optei fazer repouso, sem fazer nada. Mesmo sofrendo, via os meus outros filhos e nem dar banho neles eu podia (E8).

\section{Discussão}

A alimentação é elemento essencial no transcurso das alterações que a mulher vivencia durante o período gestacional. Essas mudanças podem ser advindas do meio externo ou interno, podendo ser modeladas pelos constructos culturais de cada uma delas. Elas entendem a gestação como um momento em que a alimentação precisa ser privilegiada (Junges, Ressel \& Monticelli, 2014), e quando há risco, como no caso das participantes deste estudo, há uma atenção reforçada com alimentação leve e fracionada, rica em nutrientes, ferro e vitaminas, com restrição de gordura, controle sódico e de carboidratos, aumento da ingesta hídrica, de frutas, verduras e alimentos integrais.

As gestantes, em especial as de alto risco, estão expostas a diversas influências do meio em que vivem. Desta forma, observa-se que a alimentação está relacionada, além dos aspectos biológicos, a fatores sociais, culturais e emocionais. As pesquisas na área biomédica preocupam-se, na maior parte, em mensurar o estado nutricional humano, por meio da análise de gráficos e de tabelas, construídos por apontamentos do ganho ou da perda de peso, da carência ou do excesso de nutrientes, entre outros valores biológicos. Esta limitação na conduta, tanto na pesquisa, quanto na assistência, implica na ausência da compreensão da totalidade de fatores que influenciam a alimentação e, principalmente, no conhecimento da história do indivíduo (Junges, Ressel \& Monticelli, 2014).

A alimentação constitui-se como uma necessidade vital para o ser humano, entretanto, ela não pode ser decidida por ninguém a não ser por ele próprio. Assim, ratifica-se a importância de o profissional de saúde conhecer os aspectos ligados à alimentação realizada pelas usuárias (o que comem, como realizam este preparo, o significado atribuído pela gestante e sua família à alimentação, por exemplo), uma vez que a aceitação de mudanças pelas gestantes de alto risco depende prioritariamente do entendimento e desejo destas.

Em algumas condições, como a anemia, a hipertensão, a diabetes e a obesidade, o controle nutricional é primordial. Uma orientação nutricional, nessa fase, é indispensável e precisa ser antecedida de uma avaliação das condições de cada gestante (Ricci, 2015). Como as práticas alimentares podem se transformar neste período, a assistência e as orientações prestadas às gestantes de alto risco precisam respeitar seus valores e crenças, de forma que elas possam confiar no profissional que as assiste, podendo refletir de forma positiva na disposição que envolve o seu próprio cuidado. 
O repouso interferiu na rotina doméstica de cuidado com a casa, com outros filhos, na vivência da sexualidade conjugal e no trabalho fora de casa. Embora necessário, este cuidado promoveu preocupação e insegurança, principalmente no que se refere ao afastamento do trabalho e as mudanças na sexualidade do casal.

Cabe destacar a necessidade das orientações serem em consonância com as necessidades humanas básicas das gestantes (Errico et al., 2018). Neste sentido, muitas vezes, os profissionais de saúde se atêm a dar as orientações necessárias, mas sem compreender como isso pode repercutir na vida das gestantes.

Assim, todas as mulheres entrevistadas referiram dificuldade para se adaptar a esta nova situação, e isso nem sempre foi percebido a contento como cuidado. Pensa-se que o enfermeiro, como importante agente de educação em saúde, e profissional que participa no pré-natal, pode atuar contextualizando o cuidado oferecido, ouvindo queixas e dificuldades de adaptação das gestantes, propondo alternativas viáveis de acordo com a realidade, e contribuindo para que elas se sintam encorajadas a realizá-lo, quando necessário.

Ademais, percebeu-se que, de certa forma, a necessidade de realizar o repouso despertou nessas mulheres sentimentos de angústia e insegurança, principalmente quando o repouso trouxe repercussões para o relacionamento sexual do casal. Em se tratando do conceito de sexualidade, atualmente, este pode ser compreendido de forma mais dinâmica e relacional. Pode ser relacionado tanto com a dimensão do ato sexual, da reprodução, da genitalidade, quanto do ponto de vista da sensualidade, do carinho, do afeto, do toque, da comunicação, da proximidade e do prazer (Figueiró, 1996), embora se perceba que, entre as participantes do estudo, esta preocupação refere-se à dimensão do ato sexual.

Destarte, o cuidado prestado pelos profissionais no pré-natal precisa atentar para que a gestante possa expressar sua sexualidade de forma segura e agradável. A abstinência sexual como prática de cuidado, pode repercutir na adesão ao cuidado e na aceitação do casal para vivenciar algumas limitações, no intuito de preservar a gestação. Porém, optar por práticas sexuais adaptativas às condições da gestação de alto risco, podem promover um estado físico e mental de completude e bem-estar (Fernández-Sola et al., 2018).

Destaca-se a necessidade do cuidado de enfermagem estar pautado na subjetividade da mulher que vivencia a gestação de alto risco, na identificação das peculiaridades bio-psico-socio-espirituais, valorizando o modo de pensar e conferindo espaços em que a troca de informações e o provimento de orientações possam ocorrer com maior efetividade (Amorim et al., 2017).

No conteúdo emocional da mulher grávida, é preciso considerar fatores psíquicos prévios e atuais, e entre os últimos, os componentes da gravidez e os fatores ambientais (Wilhelm et al., 2015; Oliveira \& Mandú, 2015). O estresse somado ao próprio risco gestacional exige atenção dos profissionais de saúde, de modo a garantir que a mulher receba um cuidado pautado na valorização dos aspectos emocionais, auxiliando-a a vivenciar a gestação de alto risco de forma saudável e tranquila.

Em contraponto, percebe-se que o componente emocional no seguimento da gestação de alto risco é quase esquecido, por receio ou desconhecimento do profissional de saúde (Wilhelm et al., 2015; Oliveira \& Mandú, 2015). Pode-se acrescentar que isso é reflexo também da formação cartesiana na área da saúde. Entretanto, pondera-se que os profissionais precisam estar atentos a qualquer sinal que possa prejudicar a vivência da gestação, envolvendo neste sentido, os aspectos emocionais, sendo fundamental orientar à mulher a se afastar do fator de estresse e formas viáveis de como lidar com isso.

Cada gestante possui valores e crenças, que repercutem nos saberes e nas práticas de cuidados realizados por elas (Alves et al., 2015). Nesse sentido, a adaptação emocional se torna relevante, pois pode ocasionar em uma diminuição na qualidade de vida, ratificando, assim, a importância de se considerar as emoções vivenciadas na gestação durante o cuidado prestado (Oliveira \& Mandú, 2015). 
É importante que o enfermeiro e os demais profissionais de saúde estejam preparados para situações, como a não adesão às práticas de cuidado, e tenham uma postura adequada, sem imposições. É preciso propor uma negociação, frente às demandas e necessidades das gestantes. Quando há riscos de complicações, a negociação no processo educativo é fundamental para auxiliar a reestruturar a vida da gestante. Nesse sentido, negociar é acordar os modelos explicativos que envolvem a paciente, a família e o profissional acerca do cuidado, por meio de propostas e contraposições na busca do entendimento (Lima et al., 2015). Portanto, depreende-se que o enfermeiro precisa exercer seu papel de cuidador, evitando posturas autoritárias e compartilhando os saberes e as práticas de cuidado com a gestante de alto risco. Assim, o respeito à cultura dos sujeitos cuidados reflete em uma assistência humanizada.

Por vezes, o profissional de saúde faz imposições às gestantes e essa atitude dificulta adesão às práticas de cuidado. Embora alguns profissionais e pacientes possuam a mesma origem cultural e social, eles percebem os problemas de saúde de maneira distinta. Cada um com suas vivências empregam diferentes sistemas de comprovação e avaliam a eficácia dos tratamentos de maneira diversa, com perspectivas diferentes (Alves et al., 2015). Logo, é imprescindível a escuta ativa dos profissionais de saúde, para que eles entendam, em primeiro lugar, as queixas e necessidades da gestante de alto risco, proporcionando entendimento e reflexão na importância da adesão às práticas de cuidados.

Cabe mencionar que as falas denotaram a preocupação que as entrevistadas tiveram para manterem sua saúde e a do bebê. Realizavam os cuidados como uma responsabilidade que superava o sofrimento da limitação que estes lhe impunham no momento. Autor da área da antropologia do cuidado refere que o sofrimento e a doença se relacionam mutuamente (Roselló, 2009). A doença, muitas vezes, leva a algum tipo de dor ou sofrimento, porém, existem sofrimentos não relacionados necessariamente à doença em si, mas aos aspectos que a envolvem (Wilhelm et al., 2015). Evidencia-se que as mulheres se sentiam responsáveis pela saúde do concepto, mas ao mesmo tempo, estavam limitadas em suas atividades cotidianas e no cuidado com os outros filhos.

O enfermeiro, na maioria das vezes, é o profissional que tem contato com a gestante de alto risco, necessitando, portanto, considerar que esta condição impõe diferentes práticas de cuidado, que podem gerar uma mobilidade emocional adicional. Durante a assistência pré-natal, ele necessita incluir ações que oportunizem a manifestação de sentimentos, pois conhecendo as reais necessidades da paciente, e entendendo-a no contexto em que vivem há maior possibilidade de auxiliá-la a minimizar as adversidades (Ortigara, Carvalho \& Pelloso, 2015).

Torna-se importante refletir acerca do sentido da gestação para a mulher e se sensibilizar quanto às vivências e peculiaridades. Numa gravidez de alto risco, se impõem cuidados especiais, que sobrecarregam emocionalmente a gestante. É importante levar em conta que uma gestação de alto risco vem acompanhada por incertezas, em um potencial maior que uma gestação sem risco obstétrico, que podem causar medo condicionado a uma responsabilização de que algo saia errado e tristeza pela situação atual (Wilhelm et al., 2015).

As práticas de cuidado que se revelaram neste estudo foram realizadas devido às necessidades especiais de uma gestação de alto risco. Condicionaram-se a partir do entendimento das gestantes de que eram necessários tais cuidados e também das orientações dos profissionais de saúde, que as acompanhavam.

Evidentemente, como os profissionais de saúde são coadjuvantes nesta experiência e desempenham importante papel para a manutenção da saúde do binômio mãe e feto, eles precisam reconhecer momentos críticos e intervir com sensibilidade, empatia e segurança, o que pode ser decisivo no bem-estar físico e emocional da gestante de alto risco (Rodrigues et al., 2016; Santos et al., 2018; Ortolani \& Ignatti, 2018). 


\section{Conclusão}

A realização deste estudo possibilitou que fossem identificadas como principais práticas de cuidado, realizadas por mulheres que vivenciaram gestação de alto risco, a alimentação balanceada e saudável, com o intuito de alcançar o sucesso no final da gestação, seguindo as orientações dos profissionais de saúde, entre eles o enfermeiro, o médico e o nutricionista; o repouso, que foi considerado como algo difícil, refletindo sentimentos de angústia e insegurança devido às alterações em suas rotinas, como o afastamento do emprego, a impossibilidade de realizar atividades que exigiam esforço físico, dentre elas, a relação sexual, o cuidado da casa e de outros filhos; e o afastamento de fatores estressantes, como algumas relações interpessoais. Os motivos que as levaram a desenvolver essas práticas de cuidado foram expressos na preocupação com a saúde do bebê e a sensação de responsabilidade pelo êxito da gestação, não sendo identificadas manifestações relacionadas ou motivadas pelos riscos quanto à saúde materna.

Frente às práticas de cuidados adotadas durante a gestação de alto risco, pode-se perceber que nem sempre foi fácil a adaptação e o seguimento dessas medidas. As mudanças na alimentação, no repouso, nas atividades diárias e, até mesmo, nas relações interpessoais denotaram em esforço para as gestantes. Assim, destaca-se a importância de o profissional de saúde estar atento à repercussão das práticas de cuidado na vida das gestantes, propondo, sempre que possível, alternativas viáveis, de acordo com a realidade de cada uma.

Espera-se que esta pesquisa forneça subsídios aos profissionais que prestam cuidado às gestantes de alto risco, mostrando a relevância de uma assistência que valorize o conhecimento das características e sentimentos singulares, as demandas originadas de suas escolhas e as experiências de cada gestante de alto risco, construindo uma relação horizontal, de apoio e motivação para com essas mulheres, de forma a permitir que se sintam seguras para vivenciar este período crítico em suas vidas. Além disso, sugere-se a construção de novas pesquisas sobre as práticas de cuidado na gestação de alto risco, bem como a compreensão dos aspectos emocionais durante esse processo, como as mulheres significam, vivenciam e conduzem suas ações frente tal experiência.

\section{Agradecimentos}

Às participantes deste estudo que vivenciaram uma gestação de alto risco e à Coordenação de Aperfeiçoamento de Pessoal de Nível Superior (CAPES) pelo apoio técnico e financeiro como bolsista do Programa de Pós-Graduação de Enfermagem da Universidade Federal de Santa Maria.

\section{Referências}

Alves, C. N. et al. (2015). Cuidado pré-natal e cultura: uma interface na atuação da enfermagem. Escola Anna Nery Revista de Enfermagem, 19(2):265-271. http://www.scielo.br/pdf/ean/v19n2/1414-8145-ean-19-02-0265.pdf

Amorim, T. V. et al. (2017). Nursing care perspectives in high-risk pregnancy: integrative review. Enfermería Global, 16(2):500. http://dx.doi.org/10.6018/eglobal.16.2.238861

Errico, L. S. P. et al. (2018). The work of nurses in high-risk prenatal care from the perspective of basic human needs. Revista Brasileira de Enfermagem, 71(suppl 3):1257-64. http://dx.doi.org/10.1590/0034-7167-2017-0328

Fernández-Sola, C. et al. (2018). Sexualidade durante todas as fases da gravidez: experiências de gestantes. Acta Paulista de Enfermagem, 31(3):305-312. http://www.scielo.br/pdf/ape/v31n3/1982-0194-ape-31-03-0305.pdf

Figueiró, M. N. D. (1996). Educação sexual: Problemas de conceituação e terminologias básicas adotadas na produção acadêmico-científica brasileira. Semina: Ciências Sociais e Humanas, 17(3):286-293.: http://www.uel.br/revistas/uel/index.php/seminasoc/article/view/9475/8267

Instituto de Pesquisa e Economia Aplicada. (2018). Metas Nacionais dos Objetivos de Desenvolvimento Sustentável.

Junges, C. F.; Ressel, L. B. \& Monticelli, M. (2014). Entre desejos e possibilidades: práticas alimentares de gestantes em uma comunidade urbana no sul do Brasil. Texto \& Contexto - Enfermagem, 23(2):382-390. https://www.scielo.br/pdf/tce/v23n2/pt_0104-0707-tce-23-02-00391.pdf

Lima, I. M., et al. (2015). High risk pregnancy: social representations of planning pregnancy, birth and family. Revista de enfermagem UFPE on line, 9(12):1255-1263. https://periodicos.ufpe.br/revistas/revistaenfermagem/article/view/10832/12029 
Research, Society and Development, v. 10, n. 1, e17710111527, 2021

(CC BY 4.0) | ISSN 2525-3409 | DOI: http://dx.doi.org/10.33448/rsd-v10i1.11527

Minayo, M. C. S. (2014). O desafio do conhecimento: pesquisa qualitativa em saúde. Hucitec.

Oliveira, D. C. \& Mandú, E. N. T. (2015). Mulheres com gravidez de maior risco: vivências e percepções de necessidades e cuidado. Escola Anna Nery Revista de Enfermagem, 19(1):93-101. http://www.scielo.br/pdf/ean/v19n1/1414-8145-ean-19-01-0093.pdf

Ortigara, E. P. F. F.; Carvalho, M. D. B. \& Pelloso, S. M. (2015). Percepção da assistência pré-natal de usuárias do serviço público de saúde. Revista de Enfermagem da UFSM, 5(4): 618-627. https://periodicos.ufsm.br/reufsm/article/view/13230/pdf

Ortolani, S.; \& Ignatti, C. (2018). Resultados da abordagem do Diabetes Mellitus Gestacional no Centro de Especialidades em Saúde da Mulher e da Criança de Itanhaém -SP. Research, Society and Development, 7 (1): 01-20, e1171122. https://www.rsdjournal.org/index.php/rsd/article/view/275/227

Resende, L. V.; Rodrigues, R. N. \& Fonseca, M. C. (2015). Mortes maternas em Belo Horizonte, Brasil: percepções sobre qualidade da assistência e evitabilidade. Revista Panamericana de Salud Pública, 37(4/5):218-224. https://www.scielosp.org/pdf/rpsp/2015.v37n4-5/218-224/pt

Ricci, S. S. (2015). Enfermagem materno-neonatal e saúde da mulher. Guanabara Koogan.

Rodrigues, A. R. M. et al. (2016). Cuidado de enfermagem na gestação de alto risco: revisão integrativa. Online Brazilian Journal of Nursing, 15(3). http://www.objnursing.uff.br/index.php/nursing/rt/printerFriendly/5434/html_2

Roselló, F. T. (2009). Antropologia do cuidar. Vozes.

Santos, L. F. et al. (2018). Características do pré-natal na perspectiva de mulheres atendidas em Unidades de Atenção Primária à Saúde. Revista de enfermagem UFPE on line, 12(2):337-44. https://periodicos.ufpe.br/revistas/revistaenfermagem/article/viewFile/230817/27812

Wilhelm, L. A. et al. (2015). Sentimentos de mulheres que vivenciaram a gestação de alto risco: estudo descritivo. Online Brazilian Journal of Nursing, 4(3). http://www.objnursing.uff.br/index.php/nursing/article/view/5206/html_882 